\title{
Perspectives on ROCK2 as a Therapeutic Target for Alzheimer's Disease
}

\author{
Audrey J. Weber and Jeremy H. Herskowitz* \\ Center for Neurodegeneration and Experimental Therapeutics, Departments of Neurology and Neurobiology, University of \\ Alabama at Birmingham, Birmingham, AL, United States
}

OPEN ACCESS

Edited by:

Qun Lu,

East Carolina University,

United States

Reviewed by:

Karen Litwa

East Carolina University,

United States

Byron Aguilar,

East Carolina University,

United States

*Correspondence: Jeremy H. Herskowitz

jhersko@uab.edu

Specialty section:

This article was submitted to

Cellular Neuropathology,

a section of the journal

Frontiers in Cellular Neuroscience

Received: 30 November 2020

Accepted: 19 February 2021

Published: 15 March 2021

Citation:

Weber AJ and Herskowitz JH (2021) Perspectives on ROCK2 as a

Therapeutic Target for Alzheimer's

Disease.

Front. Cell. Neurosci. 15:636017. doi: 10.3389/fncel.2021.636017
Rho-associated coiled-coil containing kinase isoform 2 (ROCK2) is a member of the AGC family of serine/threonine kinases and an extensively studied regulator of actinmediated cytoskeleton contractility. Over the past decade, new evidence has emerged that suggests ROCK2 regulates autophagy. Recent studies indicate that dysregulation of autophagy contributes to the development of misfolded tau aggregates among entorhinal cortex (EC) excitatory neurons in early Alzheimer's disease (AD). While the accumulation of tau oligomers and fibrils is toxic to neurons, autophagy facilitates the degradation of these pathologic species and represents a major cellular pathway for tau disposal in neurons. ROCK2 is expressed in excitatory neurons and pharmacologic inhibition of ROCK2 can induce autophagy pathways. In this mini-review, we explore potential mechanisms by which ROCK2 mediates autophagy and actin dynamics and discuss how these pathways represent therapeutic avenues for Alzheimer's disease.

Keywords: ROCK2, Alzheimer's disease, tau, autophagy, mTOR, dendritic spine

\section{INTRODUCTION}

The small Rho guanosine triphosphatase (GTPase) protein RhoA is a key regulator of various cellular processes, including actomyosin dynamics, cell proliferation, survival, and gene expression (Jaffe and Hall, 2005). The primary downstream targets of GTP-bound activated RhoA are the Rho-associated coiled-coil kinase (ROCK) isoforms 1 and 2. ROCK1 and ROCK2 are serine/threonine kinases that share $65 \%$ similarity in their amino acid sequence with 95\% similarity in their kinase domains (Nakagawa et al., 1996). Both ROCK isoforms display mostly similar expression patterns in mammals, with higher transcript levels of ROCK1 in the thymus and blood, and ROCK2 in the brain (Julian and Olson, 2014). ROCKs phosphorylate various substrates that heavily influence cellular morphology, adhesion, and motility. Through these mechanisms, ROCK1 and ROCK2 are considered therapeutic targets for cancer, asthma, insulin resistance, kidney failure, osteoporosis, and erectile dysfunction 
(Olson, 2008; Schaafsma et al., 2008; Lee et al., 2009; Albersen et al., 2010; Komers et al., 2011; Rath and Olson, 2012). In addition, ROCKs are implicated in a number of neurodegenerative disorders, including spinal cord injury, stroke, glaucoma, Alzheimer's disease (AD), Frontotemporal Dementia (FTD), Parkinson's disease, and Amyotrophic Lateral Sclerosis (ALS; Shibuya et al., 2005; Duffy et al., 2009; Herskowitz et al., 2011, 2013; Challa and Arnold, 2014; Koch et al., 2014; Gentry et al., 2016; Henderson et al., 2016; Tatenhorst et al., 2016; Gunther et al., 2017). This mini review will focus on ROCK2 and its emerging role as an autophagy regulatory protein, which may provide multiple beneficial effects for AD intervention.

\section{ROCK2 SIGNALING PROTECTS DENDRITIC SPINES IN ALZHEIMER'S DISEASE}

Dendritic spines are actin-rich protrusions along dendrites that formulate the postsynaptic sites of the majority of excitatory synapses in the brain. Spines are critical for proper neuronal function and exhibit remarkable variability in size and shape. ROCKs are well-studied regulators of actin-myosin-mediated cytoskeleton contractility, and recent work has provided evidence for ROCK isoform-specific effects on dendritic spine morphology. Two studies indicated that ROCK1 influences dendritic spine length through mechanisms involving actin-myosin pathways (Newell-Litwa et al., 2015; Henderson et al., 2019). In contrast, ROCK2 mediates spine density through the serine/threonine LIM domain kinase (LIMK) isoform 1 and subsequent phosphorylation of cofilin (Henderson et al., 2019).

Cognitive impairment in $\mathrm{AD}$ is the result of synapse loss in brain regions that are critical for memory processes. Synapse or dendritic spine loss correlates more strongly with cognitive impairment in $\mathrm{AD}$ than amyloid- $\beta$ (A $\beta)$ or neurofibrillary tangle (NFT) pathology (Braak and Braak, 1991; Scheff et al., 2006; Boros et al., 2017, 2019). This suggests that druggable targets, like ROCK2, that regulate spine density and morphology are avenues for $\mathrm{AD}$ therapeutics. To this end, $\mathrm{A} \beta$ can induce dendritic spine degeneration by activating RhoA and ROCK2. Increased activity of ROCK2 has detrimental consequences on dendritic spine structure and function in model systems (Swanger et al., 2015; Sellers et al., 2018; Henderson et al., 2019). Moreover, ROCK2 protein levels are increased among mild cognitive impairment (MCI) and $\mathrm{AD}$ patients, suggesting that ROCK2 may contribute to synaptic loss in early disease stages (Herskowitz et al., 2013). Preclinical studies demonstrated that treatment of hippocampal neurons with the pan-ROCK inhibitor Fasudil or an experimental LIMK1 inhibitor blocked $A \beta$-induced spine degeneration and neuronal hyperexcitability (Rush et al., 2018; Henderson et al., 2019). These findings highlighted the ROCK2-LIMK1 pathway as a therapeutic target to provide dendritic spine resilience against $\mathrm{A} \beta$, which could benefit cognitively normal patients that are at high risk for developing $\mathrm{AD}$ dementia. However, ROCK2 is also implicated in the intracellular degradation of the microtubule-associated protein tau via autophagic pathways. This broadens the potential benefit of ROCK2based therapies for $\mathrm{AD}$, which we will discuss later in the review.

\section{mTOR AS A REGULATOR OF AUTOPHAGY}

In eukaryotic cells, autophagy and the proteasome are major degradation pathways for intracellular debris (Mizushima and Komatsu, 2011). Autophagy is a lysosome-mediated intracellular pathway in which cytoplasmic material is delivered to and degraded in the lysosome. A major function of autophagy is to maintain quality control over proteins and organelles. Autophagy can remove long-lived, aggregated, and/or misfolded proteins, as well as regulate cellular differentiation, defense against pathogens, and nutritional starvation (Ravanan et al., 2017). Currently, three types of autophagy are known to occur in eukaryotic cells: microautophagy, chaperone-mediated autophagy, and macroautophagy. Macroautophagy is hypothesized to be the primary pathway for the delivery of cargoes to the lysosome for degradation and recycling (Hara et al., 2006; Yang and Klionsky, 2010). Henceforth, we refer to macroautophagy as "autophagy".

Autophagy uses the intermediate organelle, the autophagosome, to shuttle cytoplasmic debris to the lysosome. First, an isolation membrane, known as the phagophore, engulfs soluble materials and organelles, thereby forming the autophagosome. The autophagosome then fuses with the lysosome to become an autolysosome and degrades materials engulfed within it (Hara et al., 2006). The mammalian target of rapamycin (mTOR) is a primary upstream regulator of autophagy. mTOR is a serine/threonine kinase that forms the catalytic subunit of two distinct protein complexes, known as mTOR Complex 1 (mTORC1) and 2 (mTORC2) (Hara et al., 2006). mTORC1 has five components: mTOR, the regulatory-associated protein of $\mathrm{mTOR}$, mammalian lethal with SEC13 protein 8, proline-rich AKT1 substrate 1, and DEP domain-containing mTOR-interacting protein. mTORC2 is composed of mTOR, rapamycin-insensitive companion of mTOR (Rictor), mammalian lethal with SEC13 protein 8, and mammalian stress-activated protein kinase interacting protein 1 (Lipton and Sahin, 2014).

The induction of autophagosome biogenesis is regulated by autophagy-related (Atg) proteins that nucleate the ULK1 complex. The ULK1 complex consists of three regulatory subunits: ATG13, FIP200, and ATG101 (Saxton and Sabatini, 2017). An amino acid-rich cellular environment results in mTORC1 binding and phosphorylating ULK1 at Ser757, resulting in the inhibition of autophagosome formation (Ganley et al., 2009; Jung et al., 2009). During amino acid starvation, mTORC1 dissociates from ULK1, thereby allowing ULK1 activation by AMP-activated protein kinase (AMPK), a key activator of autophagosome formation (Kim et al., 2011). In an alternative pathway, activation and translocation of the transcription factor EB (TFEB) into the nucleus leads to increased expression of genes related to lysosomal biogenesis 
and autophagy machinery (Martina et al., 2012; RoczniakFerguson et al., 2012; Settembre et al., 2012). mTORC1 can also regulate autophagy in part by phosphorylating TFEB. TFEB phosphorylation at Ser211 results in the inactivation of TFEB in the cytoplasm (Martina et al., 2012).

mTORC2 is known to phosphorylate members of the AGC family of protein kinases, including protein kinase B (also known as Akt), protein kinase $\mathrm{C}$, and serum and glucocorticoid-regulated kinase 1 (SGK1) (Saxton and Sabatini, 2017). Phosphorylation of Akt by mTORC2 may play dual roles concerning autophagy induction. One study suggests that phosphorylation of Akt by mTORC2 inhibits the activation of the transcription factor FoxO3, thereby suppressing autophagy-related gene expression (Mammucari et al., 2007). Recent work evaluating loss of mTORC2 activity showed that the downstream effector SGK-1 can inhibit autophagy independent of FoxO3 (Aspernig et al., 2019). Additional work in primary rat hippocampal neurons demonstrated that mTORC2mediated phosphorylation of Akt can activate mTORC1, thus influencing autophagy through an alternative mechanism (Urbanska et al., 2012). mTORC2-mediated activation of mTORC1 was further confirmed in human cells, which showed a reduction of phosphorylated mTORC1 and p70S6K, indicating mTORC2 can inhibit autophagy through activation of mTORC1 (Huang et al., 2015). Collectively, these studies indicate that both mTORC1 and mTORC2 can modulate autophagy induction. However, mTORC2 is also an intriguing regulator of the actin cytoskeleton, which we will discuss later in the review.

\section{AUTOPHAGY IN ALZHEIMER'S DISEASE}

$\mathrm{AD}$ neuropathology is characterized by the presence of extracellular $A \beta$ deposits and intracellular NFTs comprised of misfolded, hyperphosphorylated tau (Wu et al., 2016; Goedert et al., 2017). The pathological spread of tau aggregates correlates with cognitive decline in $\mathrm{AD}$, suggesting that propagation of NFTs contributes to synapse loss (Braak et al., 2006; Scholl et al., 2016). The spread of tau in AD is hypothesized to operate in a prion-like manner in which tau seeds spread via connected neurons. The tau seed(s) is hypothesized to interact with native tau in the cytosol, drive misfolding of tau, and ultimately generate neurofibrillary pathology (Hallinan et al., 2019). In the early stages of $\mathrm{AD}$, tau aggregates are found in somatodendritic compartments of layer $2 / 3$ neurons in the entorhinal cortex (EC) (Braak and Braak, 1991). It is hypothesized that pathologic tau propagates from the EC to the hippocampus via trans-synaptic connections, where it can spread to limbic regions and ultimately neocortical areas in the same manner (Braak and Braak, 1991; De Calignon et al., 2012; Ahmed et al., 2014; Figure 1A).

The precise molecular and cellular mechanisms of tau propagation remain critical questions in the field, but the selective vulnerability of neurons in EC layer $2 / 3$, the subiculum, CA1 of the hippocampus, and basal forebrain cholinergic neurons likely contributes to the initiation of tau aggregates (Davies and Maloney, 1976; Hyman et al., 1984; Stranahan and Mattson, 2010). A recent study to understand the selective vulnerability of neurons suggests that excitatory neurons, in contrast to inhibitory neurons, harbor a cellular environment of aggregation-prone proteins that is susceptible to dysregulation of protein homeostasis systems (Fu et al., 2019). Co-expression network analysis of single-nucleus RNA-seq datasets identified the autophagy facilitator BCL2-associated athanogene 3 (BAG3) as a protector from tau aggregation. Notably, inhibitory neurons, which are often thought to be resilient to tau aggregation, have high expression of BAG3 in both $\mathrm{AD}$ and non-AD cases (Fu et al., 2019).

Dysfunction of autophagy and the lysosome system is hypothesized to contribute to $\mathrm{AD}$ progression (Boland et al., 2008). Possession of the $\varepsilon 4$ allele of Apolipoprotein E (APOE4), a major genetic risk factor for the development of late-onset $\mathrm{AD}$, was shown to disrupt the endo-lysosomal system in EC neurons (Nuriel et al., 2017). Transcriptomic analysis of APOE3/4 targeted replacement mice identified upregulation of genes associated with vesicle function including nine Vacuolar $\mathrm{H}^{+}$ATPases (V-ATPases) and Rab GTPases which are vital for early and late endosome function. Moreover, immunohistochemistry of the EC and cingulate cortex of APOE4/4 targeted replacement mice revealed an increase of early endosomes, which is observed in AD (Nuriel et al., 2017). The dysregulation of autophagy within the EC could facilitate the initial aggregation of tau that ultimately spawns pathologic tau spread to other vulnerable brain regions.

To this end, studies in post-mortem $\mathrm{AD}$ patient tissue revealed accumulation of autophagic vacuoles within damaged neurites, suggesting defective lysosomal clearance in the neocortex (Nixon et al., 2005). Other studies indicated an increase in mTOR signaling activity in AD brains (Chang et al., 2002; Peel, 2003; Onuki et al., 2004). Immunoblot findings from Tramutola et al. (2015) revealed that mTOR phosphorylation was increased in both $\mathrm{AD}$ and mild cognitive impairment (MCI) cases, but not in preclinical $\mathrm{AD}$ brains. Furthermore, two autophagy-related proteins, Beclin1 and LC3, were decreased in both $\mathrm{AD}$ and $\mathrm{MCI}$ tissue, suggesting that mTOR activity-induced autophagy was impaired (Tramutola et al., 2015). These findings support the hypothesis that an increase in mTOR signaling activity could suppress autophagyrelated processes, leading to a failure to clear tau aggregates in vulnerable neurons.

From a preclinical standpoint, rapamycin-induced inhibition of mTOR in the $3 \mathrm{xTg}$-AD mouse model increased the abundance of autophagy-related proteins, while simultaneously reducing both soluble $A \beta$ and tau levels (Caccamo et al., 2010). Recent work in cultured human neurons derived from FTD patients further demonstrated a role for mTOR inhibition as a therapeutic strategy to reduce tau levels (Silva et al., 2020). In this study, small molecular screens were used to identify ATP-competitive mTOR inhibitors that are more selective and potent than rapamycin. Patient-derived human neurons were treated with mTOR inhibitors, resulting in decreased levels of insoluble tau after a single dosage. The treatment had lasting effects by increasing autophagy for 12 days post-drug administration. Furthermore, mTOR inhibitors increased neuronal viability by protecting against tau toxicity and stressors such as $A \beta$ (Silva 
et al., 2020). Together, these findings suggest that mitigating pathogenic tau levels by activating autophagic pathways is a rational strategy for treating tauopathies, including $\mathrm{AD}$. As discussed earlier in this review, pharmacologic inhibition of ROCK2 signaling pathways can generate dendritic spine resilience to $\mathrm{A} \beta$ by stimulating actin cytoskeleton rearrangement. However, ROCK2 inhibition can also reduce tau levels by inducing autophagy. This fascinating combination of beneficial effects highlights the potential of ROCK2-based therapeutics for $\mathrm{AD}$. Yet, important questions remain regarding the potential intersection of these two pathways.

\section{ROCK2 AS A THERAPEUTIC TARGET FOR ALZHEIMER'S DISEASE}

Because the spread of NFTs, indicated by increasing Braak stages (Figure 1A), correlates more strongly with cognitive decline in $\mathrm{AD}$ than extracellular $\mathrm{A} \beta$ pathology, it is hypothesized that propagation of tau aggregates contributes to the dendritic spine or synapse loss (Braak et al., 2006; Scholl et al., 2016). Based on this, therapeutics that curb tau propagation, as well as spine loss, would be considered a rational treatment strategy to delay or prevent $\mathrm{AD}$ dementia onset. Notably, the hypotensive effect of orally available pan-ROCK inhibitors is likely linked to ROCK1 inhibition, and therefore ROCK2-specific compounds could offer a less severe drop in blood pressure upon systemic exposure (Defert and Boland, 2017). ROCK2's emerging role as an autophagy regulatory protein, as well as ROCK2's established link to actin cytoskeleton dynamics, positions it as an exciting, highly interesting drug target. The pan-ROCK inhibitor Fasudil has been clinically approved to treat hypertension, heart failure, glaucoma, spinal cord injury, and stroke (Feng et al., 2015). Based on the beneficial effects of Fasudil on neuronal plasticity and survival, Fasudil is currently being used in clinical trials for the treatment of ALS (Lingor et al., 2019). These efforts fuel excitement about the potential of ROCK inhibitors for the treatment of other neurodegenerative disorders, including AD.

$\mathrm{A} \beta$, a key pathological hallmark of $\mathrm{AD}$, is generated by sequential proteolytic cleavage of the amyloid precursor protein (APP). Fasudil, as well as the experimental smallmolecule inhibitor SR3677, which is more selective to ROCK2, were shown to reduce $A \beta$ production in primary neurons and a mouse model of AD. Cell biological studies in HEK293 cells indicated that treatment with SR3677 increased APP and beta-site cleaving enzyme 1 (BACE1) co-localization with the lysosome marker LAMP1, suggesting that pharmacologic inhibition of ROCK2 promotes traffic of APP and BACE1 to lysosomes (Herskowitz et al., 2013). Further studies indicated that RNAi depletion of ROCK1 or ROCK2 in primary neurons reduced endogenous murine $\mathrm{A} \beta$ production, which was likely the result of enhanced lysosomal or autophagic degradation of APP (Henderson et al., 2016).

Several studies indicate that pharmacologic inhibition of ROCKs can induce autophagy in mammalian cells (Bauer et al., 2009; Koch et al., 2014; Gentry et al., 2016). Using

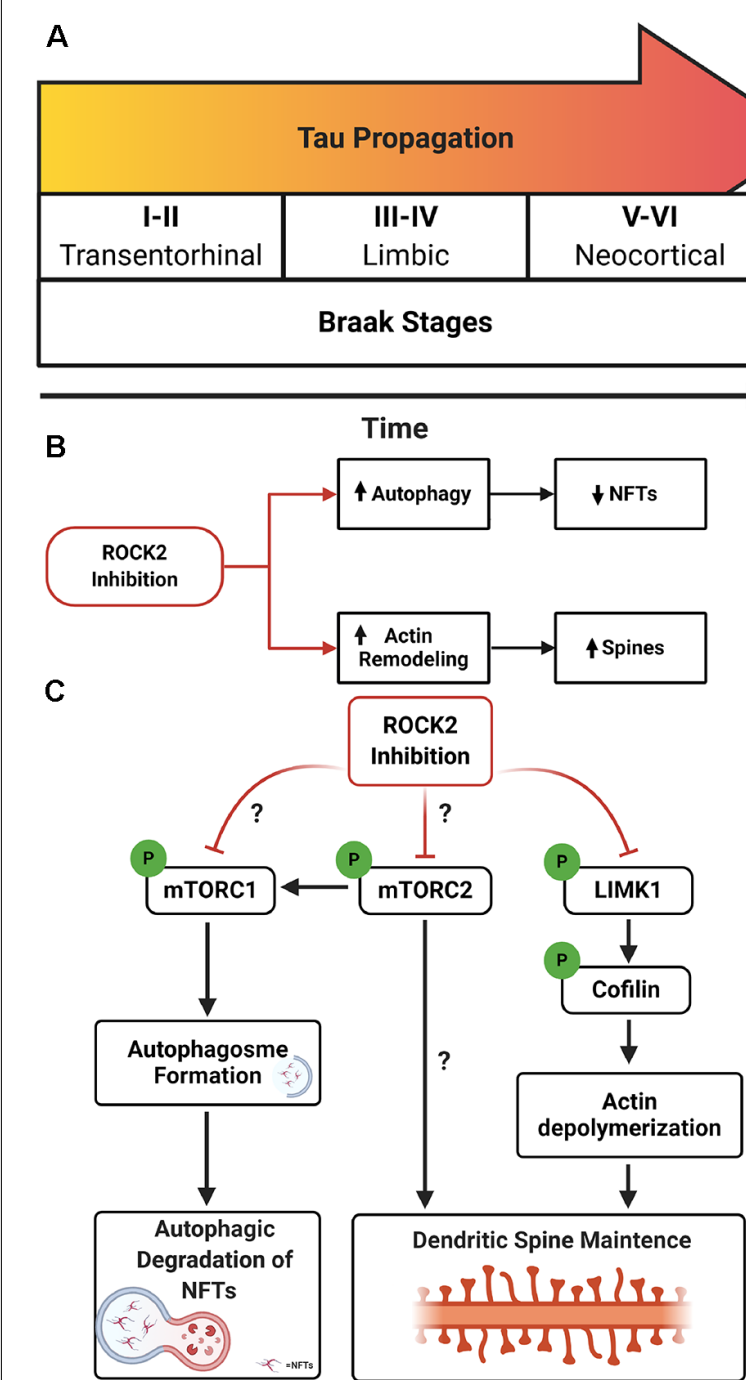

FIGURE 1 | Rho-associated coiled-coil kinase isoforms 2 (ROCK2) inhibition as a therapeutic avenue for Alzheimer's disease (AD). (A) Schematic of Braak stages I-VI which describes the hypothetical spread of neurofibrillary tangles (NFTs) over time in AD. Tau aggregates initiate in the entorhinal cortex (EC; stages I-II) before spreading to limbic regions such as the hippocampus (stages III-IV) and eventually the neocortex (stages V-VI). (B) Therapeutic benefits of targeting ROCK2 in AD. Pharmacologic inhibition of ROCK2 enhances autophagic processes, reducing tau protein levels and pathology. In parallel, ROCK2 inhibition can increase actin dynamics, stimulating dendritic spine structural plasticity. (C) Mechanisms of ROCK2 therapeutics. (Left to right) ROCK2 inhibition reduces mammalian target of rapamycin (mTOR) phosphorylation which stimulates autophagosome formation and facilitates tau aggregate degradation. Reducing mTOR phosphorylation could influence the activity of mTORC1 and/or mTORC2. ROCK2 may alter mTORC2's role in actin cytoskeleton rearrangement. ROCK2 inhibition reduces phosphorylation of LIMK1, inactivating LIMK1. Reduced activity of LIMK1 maintains cofilin activation and actin depolymerization, enhancing structural plasticity of dendritic spines.

RNAi depletion methods, Koch et al. (2014) demonstrated that knockdown of ROCK2 or LIMK1 enhanced neurite outgrowth of rat retinal ganglion cells (RGCs) following rat optic nerve crush. However, knockdown of ROCK2, but 
not LIMK1, increased survival of RGCs after optic nerve axotomy. Further shRNA-mediated knockdown studies of ROCK2 in primary RGC cultures revealed that depletion of ROCK2 increased protein levels of the microtubuleassociated protein 1 light chain 3 (LC3-II), which is an essential component for autophagosome formation. Moreover, SQSTM1/p62, which serves as a link between LC3 and ubiquitinated substrates, was reduced after ROCK2 depletion (Koch et al., 2014). Collectively, these findings suggest that inhibition of ROCK2 kinase activity enhances autophagic flux and that this mechanism does not rely on ROCK2 signaling through LIMK1.

Notably, ROCK1 can play a role in metabolic stress-induced autophagy. ROCK1 activity induces autophagy in HeLa cells by interacting with and phosphorylating Beclin1 at threonine 119, whereas blocking ROCK1 activity with the pan-ROCK inhibitor Y27632 reduced nutritional stress-mediated autophagy (Gurkar et al., 2013). In contrast, genetic deletion of ROCK1 had no effect on mTOR signaling in cardiomyocytes (Shi et al., 2019). These findings suggest that ROCK1's influence over autophagic pathways may be cell-type specific, and whether these mechanisms exist in brain-derived cells remains to be determined.

Postmortem studies in progressive supranuclear palsy (PSP) and corticobasal degeneration (CBD) patients revealed that ROCK1, ROCK2, mTOR, and p70 S6 kinase (S6K) protein levels are increased significantly in dorsolateral prefrontal cortex tissue samples from PSP and CBD cases compared with controls. Moreover, statistical correlation analysis indicated a strong positive correlation among ROCK1, ROCK2, mTOR, and S6K in PSP and CBD cases, suggesting that these signaling pathways could play a role in tau deposition in disease (Gentry et al., 2016). Notably, postmortem studies on MCI and AD patients indicated ROCK2 protein levels are increased in neurons from the dorsolateral prefrontal cortex among disease cases in comparison to controls (Herskowitz et al., 2013). However, whether similar changes in mTOR and S6K protein levels occur in the prefrontal cortex among all tauopathies, including $\mathrm{AD}$, remains to be determined.

Disposal of tau can also be mediated by autophagy, specifically via mTOR signaling through S6K in neurons (Boland et al., 2008; Kruger et al., 2012). Neurons treated with SR3677 exhibited robust depletion of soluble and insoluble tau protein levels which coincided with reduced mTOR phosphorylation at serine 2448, reduced protein levels of S6K and p62, and increased protein levels of LC3-II (Gentry et al., 2016). These findings indicate that ROCK2 inhibition stimulates autophagy induction in neurons and results in the reduction of tau protein levels. Notably, Gentry et al. also showed that treatment with Fasudil mitigated pathogenic tau levels and suppressed rough eye phenotype in a Drosophila model of tauopathy by inducing autophagic pathways (Gentry et al., 2016). The evidence suggests that ROCK2 inhibition would have beneficial effects on both structural plasticities of neurons, through ROCK2's prominent role in actin dynamics, and autophagic clearance of tau in $\mathrm{AD}$ (Figure 1B). Yet, critical questions remain regarding the precise molecular mechanisms involved and the putative interactions of ROCK2 signaling and mTOR pathways.

RNAi depletion of drug inhibition of ROCK2 reduces mTOR phosphorylation (Gentry et al., 2016); therefore, ROCK2 could influence autophagic pathways through mTORC1 and/or mTORC2. Whether ROCK2 signaling preferentially affects the mTORC1 or mTORC2 pathway remains to be determined. Moreover, mTORC2 can regulate autophagy through Akt which in turn mediates mTORC1 activity (Huang et al., 2015), thus ROCK2 could alter mTORC1 pathways through mTORC2. Finally, one of the most intriguing possibilities is that ROCK2 could regulate actin cytoskeleton dynamics by influencing mTORC2 activity (Figure 1C).

There is strong evidence in neurons that ROCK2 controls actin cytoskeleton rearrangement and dendritic structure through LIMK1 and cofilin (Rush et al., 2018; Henderson et al., 2019). However, could ROCK2 have similar effects on dendritic morphology by modulating mTORC2 activity? While mTORC2 does not seemingly affect organ size in skeletal muscle (Bentzinger et al., 2008), adipose tissue (Cybulski et al., 2009), or kidney (Gödel et al., 2011), brain architecture and neuron size are affected, suggesting a role for mTORC2 in CNS morphology (Angliker and Rüegg, 2013; Thomanetz et al., 2013). Conditional deletion of Rictor, a key component of mTORC2, in forebrain excitatory neurons of mice lead to decreased Ras-related C3 botulinum toxin substrate 1 (Rac1)-GTPase activity and reduced phosphorylation of downstream targets, including p21-activated kinase (PAK) and cofilin (Huang et al., 2013). This intersection of mTORC2 and Rho GTPase signaling could reflect a connection between ROCK2 and mTORC2 concerning actin re-organization in neurons, however additional experiments are warranted to fully elucidate the relationship. ROCK2's emerging role as an autophagy regulatory protein, functioning through mTOR-related pathways, suggests exciting avenues of therapeutic potential for suppressing tau propagation in $\mathrm{AD}$.

Work on the roles of ROCK1 and ROCK2 in non-neuronal cells in the brain has been more limited compared to neuron-centric studies. Not surprisingly, microarray and subsequent bioinformatics analyses of cultured primary murine astrocytes treated with the pan-ROCK inhibitor Fasudil demonstrated that inhibition of ROCKs altered transcription profiles associated with astrocytic morphology and motility, including genes involved in the actin cytoskeleton, extracellular matrix, and tight junctions. However, a portion of gene transcription alterations revealed that Fasudil induced pro-survival phenotypes in astrocytes that included excitatory amino acid transporter 2 and brain-derived neurotrophic factor as well as metabolic and anti-oxidative gene clusters (Lau et al., 2012). To this end, preclinical studies using Fasudil in rodent models of amyotrophic lateral sclerosis indicated that Fasudil treatment reduced infiltration of astrocytes but increased the presence of Ibal-positive microglial cells to sites of spinal cord degeneration. Parallel in vitro studies of microglial treated with lipopolysaccharides showed that Fasudil reduced secretion of proinflammatory cytokines and chemokines, including tumor necrosis factor- $\alpha$, 
interleukin 6, and chemokine (C-C motif) ligands 2, 3 and 5 (Tonges et al., 2014). Additional work will be critical to better understand if and/or how ROCKs may affect autophagic pathways in non-neuronal cells in the brain.

\section{AUTHOR CONTRIBUTIONS}

AJW and JHH wrote the article. All authors contributed to the article and approved the submitted version.

\section{REFERENCES}

Ahmed, Z., Cooper, J., Murray, T. K., Garn, K., Mcnaughton, E., Clarke, H., et al. (2014). A novel in vivo model of tau propagation with rapid and progressive neurofibrillary tangle pathology: the pattern of spread is determined by connectivity, not proximity. Acta Neuropathol. 127, 667-683. doi: 10.1007/s00401-014-1254-6

Albersen, M., Shindel, A. W., Mwamukonda, K. B., and Lue, T. F. (2010). The future is today: emerging drugs for the treatment of erectile dysfunction. Expert. Opin. Emerg. Drugs 15, 467-480. doi: 10.1517/14728214.2010.480973

Angliker, N., and Rüegg, M. A. (2013). In vivo evidence for mTORC2-mediated actin cytoskeleton rearrangement in neurons. Bioarchitecture 3, 113-118. doi: 10.4161/bioa.26497

Aspernig, H., Heimbucher, T., Qi, W., Gangurde, D., Curic, S., Yan, Y., et al. (2019). Mitochondrial perturbations couple mtorc2 to autophagy in C. elegans. Cell Rep. 29, e5.1399-e5.1409. doi: 10.1016/j.celrep.2019.09.072

Bauer, P. O., Wong, H. K., Oyama, F., Goswami, A., Okuno, M., Kino, Y., et al. (2009). Inhibition of rho kinases enhances the degradation of mutant huntingtin. J. Biol. Chem. 284, 13153-13164. doi: 10.1074/jbc.M809229200

Bentzinger, C. F., Romanino, K., Cloëtta, D., Lin, S., Mascarenhas, J. B., Oliveri, F., et al. (2008). Skeletal muscle-specific ablation of raptor, but not of rictor, causes metabolic changes and results in muscle dystrophy. Cell Metab. 8, 411-424. doi: 10.1016/j.cmet.2008.10.002

Boland, B., Kumar, A., Lee, S., Platt, F. M., Wegiel, J., Yu, W. H., et al. (2008). Autophagy induction and autophagosome clearance in neurons: relationship to autophagic pathology in Alzheimer's disease. J. Neurosci. 28, 6926-6937. doi: 10.1523/JNEUROSCI.0800-08.2008

Boros, B. D., Greathouse, K. M., Gearing, M., and Herskowitz, J. H. (2019). Dendritic spine remodeling accompanies Alzheimer's disease pathology and genetic susceptibility in cognitively normal aging. Neurobiol. Aging 73, 92-103. doi: 10.1016/j.neurobiolaging.2018.09.003

Boros, B. D., Greathouse, K. M., Gentry, E. G., Curtis, K. A., Birchall, E. L., Gearing, M., et al. (2017). Dendritic spines provide cognitive resilience against Alzheimer's disease. Ann. Neurol. 82, 602-614. doi: 10.1002/ana.25049

Braak, H., Alafuzoff, I., Arzberger, T., Kretzschmar, H., and Del Tredici, K. (2006). Staging of Alzheimer disease-associated neurofibrillary pathology using paraffin sections and immunocytochemistry. Acta Neuropathol. 112, 389-404. doi: 10.1007/s00401-006-0127-z

Braak, H., and Braak, E. (1991). Neuropathological stageing of Alzheimer-related changes. Acta Neuropathol. 82, 239-259. doi: 10.1007/BF00308809

Caccamo, A., Majumder, S., Richardson, A., Strong, R., and Oddo, S. (2010). Molecular interplay between mammalian target of rapamycin (mTOR), amyloid- $\beta$ and tau: effects on cognitive impairments. J. Biol. Chem. 285, 13107-13120. doi: 10.1074/jbc.M110.100420

Challa, P., and Arnold, J. J. (2014). Rho-kinase inhibitors offer a new approach in the treatment of glaucoma. Expert. Opin. Investig. Drugs 23, 81-95. doi: $10.1517 / 13543784.2013 .840288$

Chang, R. C. C., Wong, A. K. Y., Ng, H. -K., and Hugon, J. (2002). Phosphorylation of eukaryotic initiation factor- $2 \alpha$ (eIF2 $\alpha$ ) is associated with neuronal degeneration in Alzheimer's disease. NeuroReport 13, 2429-2432. doi: 10.1097/00001756-200212200-00011

Cybulski, N., Polak, P., Auwerx, J., Rüegg, M. A., and Hall, M. N. (2009). mTOR complex 2 in adipose tissue negatively controls whole-body growth. Proc. Natl. Acad. Sci. U S A 106, 9902-9907. doi: 10.1073/pnas.0811321106

\section{FUNDING}

This work was supported by the National Institutes of Health through the National Institute of Aging AG061800, AG054719, and AG063755 to JHH.

\section{ACKNOWLEDGMENTS}

We thank Courtney $\mathrm{K}$. Walker for the critical reading of this manuscript.

Davies, P., and Maloney, A. J. (1976). Selective loss of central cholinergic neurons in Alzheimer's disease. Lancet 2:1403. doi: 10.1016/s0140-6736(76)91936-X

De Calignon, A., Polydoro, M., Suárez-Calvet, M., William, C., Adamowicz, D. H., Kopeikina, K. J., et al. (2012). Propagation of tau pathology in a model of early Alzheimer's disease. Neuron 73, 685-697. doi: 10.1016/j.neuron.2011.11.033

Defert, O., and Boland, S. (2017). Rho kinase inhibitors: a patent review (2014 2016). Expert. Opin. Ther. Patents 27, 507-515. doi: 10.1080/13543776.2017. 1272579

Duffy, P., Schmandke, A., Sigworth, J., Narumiya, S., Cafferty, W. B., and Strittmatter, S. M. (2009). Rho-associated kinase II (ROCKII) limits axonal growth after trauma within the adult mouse spinal cord. J. Neurosci. 29, 15266-15276. doi: 10.1523/JNEUROSCI.4650-09.2009

Feng, Y., Lograsso, P. V., Defert, O., and Li, R. (2015). Rho kinase (ROCK) inhibitors and their therapeutic potential. J. Med. Chem. 59, 2269-2300. doi: 10.1021/acs.jmedchem.5b00683

Fu, H., Possenti, A., Freer, R., Nakano, Y., Hernandez Villegas, N. C., Tang, M., et al. (2019). A tau homeostasis signature is linked with the cellular and regional vulnerability of excitatory neurons to tau pathology. Nat. Neurosci. 22, 47-56. doi: 10.1038/s41593-018-0298-7

Ganley, I. G., Lam Du, H., Wang, J., Ding, X., Chen, S., and Jiang, X. (2009). ULK1.ATG13.FIP200 complex mediates mTOR signaling and is essential for autophagy. J. Biol. Chem. 284, 12297-12305. doi: 10.1074/jbc.M900573200

Gentry, E. G., Henderson, B. W., Arrant, A. E., Gearing, M., Feng, Y., Riddle, N. C., et al. (2016). Rho kinase inhibition as a therapeutic for progressive supranuclear palsy and corticobasal degeneration. J. Neurosci. 36, 1316-1323. doi: 10.1523/JNEUROSCI.2336-15.2016

Gödel, M., Hartleben, B., Herbach, N., Liu, S., Zschiedrich, S., Lu, S., et al. (2011). Role of mTOR in podocyte function and diabetic nephropathy in humans and mice. J. Clin. Invest. 121, 2197-2209. doi: 10.1172/JCI44774

Goedert, M., Eisenberg, D. S., and Crowther, R. A. (2017). Propagation of tau aggregates and neurodegeneration. Annu. Rev. Neurosci. 40, 189-210. doi: 10.1146/annurev-neuro-072116-031153

Gunther, R., Balck, A., Koch, J. C., Nientiedt, T., Sereda, M., Bahr, M., et al. (2017). Rho kinase inhibition with fasudil in the SOD1(G93A) mouse model of amyotrophic lateral sclerosis-symptomatic treatment potential after disease onset. Front. Pharmacol. 8:17. doi: 10.3389/fphar.2017.00017

Gurkar, A. U., Chu, K., Raj, L., Bouley, R., Lee, S. H., Kim, Y. B., et al. (2013). Identification of ROCK1 kinase as a critical regulator of beclin1-mediated autophagy during metabolic stress. Nat. Commun. 4:2189. doi: $10.1038 /$ ncomms3189

Hallinan, G. I., Vargas-Caballero, M., West, J., and Deinhardt, K. (2019). Tau misfolding efficiently propagates between individual intact hippocampal neurons. J. Neurosci. 39, 9623-9632. doi: 10.1523/JNEUROSCI.1590-19.2019

Hara, T., Nakamura, K., Matsui, M., Yamamoto, A., Nakahara, Y., SuzukiMigishima, R., et al. (2006). Suppression of basal autophagy in neural cells causes neurodegenerative disease in mice. Nature 441, 885-889. doi: $10.1038 /$ nature 04724

Henderson, B. W., Gentry, E. G., Rush, T., Troncoso, J. C., Thambisetty, M., Montine, T. J., et al. (2016). Rho-associated protein kinase 1 (ROCK1) is increased in Alzheimer's disease and ROCK1 depletion reduces amyloid-beta levels in brain. J. Neurochem. 138, 525-531. doi: 10.1111/jnc. 13688

Henderson, B. W., Greathouse, K. M., Ramdas, R., Walker, C. K., Rao, T. C., Bach, S. V., et al. (2019). Pharmacologic inhibition of LIMK1 provides 
dendritic spine resilience against beta-amyloid. Sci. Signal. 12:eaaw9318. doi: 10.1126/scisignal.aaw9318

Herskowitz, J. H., Feng, Y., Mattheyses, A. L., Hales, C. M., Higginbotham, L. A., Duong, D. M., et al. (2013). Pharmacologic inhibition of ROCK2 suppresses amyloid-beta production in an Alzheimer's disease mouse model. J. Neurosci. 33, 19086-19098. doi: 10.1523/JNEUROSCI.2508-13.2013

Herskowitz, J. H., Seyfried, N. T., Gearing, M., Kahn, R. A., Peng, J., Levey, A. I., et al. (2011). Rho kinase II phosphorylation of the lipoprotein receptor LR11/SORLA alters amyloid-beta production. J. Biol. Chem. 286, 6117-6127. doi: 10.1074/jbc.M110.167239

Huang, N., Wu, J., Qiu, W., Lyu, Q., He, J., Xie, W., et al. (2015). MiR-15a and miR-16 induce autophagy and enhance chemosensitivity of Camptothecin. Cancer Biol. Ther. 16, 941-948. doi: 10.1080/15384047.2015.10 40963

Huang, W., Zhu, P. J., Zhang, S., Zhou, H., Stoica, L., Galiano, M., et al. (2013). mTORC2 controls actin polymerization required for consolidation of long-term memory. Nat. Neurosci. 16, 441-448. doi: 10.1038/nn.3351

Hyman, B. T., Van Hoesen, G. W., Damasio, A. R., and Barnes, C. L. (1984). Alzheimer's disease: cell-specific pathology isolates the hippocampal formation. Science 225, 1168-1170. doi: 10.1126/science.6474172

Jaffe, A. B., and Hall, A. (2005). Rho GTPases: biochemistry and biology. Annu. Rev. Cell Dev. Biol. 21, 247-269. doi: 10.1146/annurev.cellbio.21.020604. 150721

Julian, L., and Olson, M.F. (2014). Rho-associated coiled-coil containing kinases (ROCK): structure, regulation, and functions. Small GTPases 5:e29846. doi: $10.4161 /$ sgtp. 29846

Jung, C. H., Jun, C. B., Ro, S. H., Kim, Y. M., Otto, N. M., Cao, J., et al. (2009). ULK-Atg13-FIP200 complexes mediate mTOR signaling to the autophagy machinery. Mol. Biol. Cell 20, 1992-2003. doi: 10.1091/mbc.e08-12-1249

Kim, J., Kundu, M., Viollet, B., and Guan, K. L. (2011). AMPK and mTOR regulate autophagy through direct phosphorylation of Ulk1. Nat. Cell Biol. 13, 132-141. doi: $10.1038 /$ ncb2152

Koch, J. C., Tonges, L., Barski, E., Michel, U., Bahr, M., and Lingor, P. (2014). ROCK2 is a major regulator of axonal degeneration, neuronal death and axonal regeneration in the CNS. Cell Death Dis. 5:e1225. doi: 10.1038/cddis.2014.191

Komers, R., Oyama, T. T., Beard, D. R., Tikellis, C., Xu, B., Lotspeich, D. F., et al. (2011). Rho kinase inhibition protects kidneys from diabetic nephropathy without reducing blood pressure. Kidney Int. 79, 432-442. doi: 10.1038/ki. 2010.428

Kruger, U., Wang, Y., Kumar, S., and Mandelkow, E. M. (2012). Autophagic degradation of tau in primary neurons and its enhancement by trehalose. Neurobiol. Aging 33, 2291-2305. doi: 10.1016/j.neurobiolaging.2011.11.009

Lau, C. L., Perreau, V. M., Chen, M. J., Cate, H. S., Merlo, D., Cheung, N. S., et al. (2012). Transcriptomic profiling of astrocytes treated with the Rho kinase inhibitor fasudil reveals cytoskeletal and pro-survival responses. J. Cell Physiol. 227, 1199-1211. doi: $10.1002 /$ jcp.22838

Lee, D. H., Shi, J., Jeoung, N. H., Kim, M. S., Zabolotny, J. M., Lee, S. W., et al. (2009). Targeted disruption of ROCK1 causes insulin resistance in vivo. J. Biol. Chem. 284, 11776-11780. doi: 10.1074/jbc.C900014200

Lingor, P., Weber, M., Camu, W., Friede, T., Hilgers, R., Leha, A., et al. (2019). ROCK-ALS: protocol for a randomized, placebo-controlled, doubleblind phase iia trial of safety, tolerability and efficacy of the rho kinase (rock) inhibitor fasudil in amyotrophic lateral sclerosis. Front. Neurol. 10:293. doi: 10.3389/fneur.2019.00293

Lipton, J. O., and Sahin, M. (2014). The neurology of mTOR. Neuron 84, 275-291. doi: 10.1016/j.neuron.2014.09.034

Mammucari, C., Milan, G., Romanello, V., Masiero, E., Rudolf, R., Del Piccolo, P., et al. (2007). FoxO3 controls autophagy in skeletal muscle in vivo. Cell Metab. 6, 458-471. doi: 10.1016/j.cmet.2007.11.001

Martina, J. A., Chen, Y., Gucek, M., and Puertollano, R. (2012). MTORC1 functions as a transcriptional regulator of autophagy by preventing nuclear transport of TFEB. Autophagy 8, 903-914. doi: 10.4161/auto.19653

Mizushima, N., and Komatsu, M. (2011). Autophagy: renovation of cells and tissues. Cell 147, 728-741. doi: 10.1016/j.cell.2011.10.026

Nakagawa, O., Fujisawa, K., Ishizaki, T., Saito, Y., Nakao, K., and Narumiya, S. (1996). ROCK-I and ROCK-II, two isoforms of rho-associated coiled-coil forming protein serine/threonine kinase in mice. FEBS. Lett. 392, 189-193. doi: 10.1016/0014-5793(96)00811-3
Newell-Litwa, K. A., Badoual, M., Asmussen, H., Patel, H., Whitmore, L., and Horwitz, A. R. (2015). ROCK1 and 2 differentially regulate actomyosin organization to drive cell and synaptic polarity. J. Cell Biol. 210, 225-242. doi: $10.1083 /$ jcb. 201504046

Nixon, R. A., Wegiel, J., Kumar, A., Yu, W. H., Peterhoff, C., Cataldo, A., et al. (2005). Extensive involvement of autophagy in Alzheimer disease: an immuno-electron microscopy study. J. Neuropathol. Exp. Neurol. 64, 113-122. doi: $10.1093 /$ jnen/64.2.113

Nuriel, T., Peng, K.Y., Ashok, A., Dillman, A. A., Figueroa, H. Y., Apuzzo, J., et al. (2017). The endosomal-lysosomal pathway is dysregulated by APOE4 expression in vivo. Front. Neurosci. 11:702. doi: 10.3389/fnins. 2017.00702

Olson, M. F. (2008). Applications for ROCK kinase inhibition. Curr. Opin. Cell Biol. 20, 242-248. doi: 10.1016/j.ceb.2008.01.002

Onuki, R., Bando, Y., Suyama, E., Katayama, T., Kawasaki, H., Baba, T., et al. (2004). An RNA-dependent protein kinase is involved in tunicamycin-induced apoptosis and Alzheimer's disease. EMBO J. 23, 959-968. doi: 10.1038/sj.emboj. 7600049

Peel, A. (2003). Activation of the cell stress kinase PKR in Alzheimer's disease and human amyloid precursor protein transgenic mice. Neurobiol. Dis. 14, 52-62. doi: 10.1016/s0969-9961(03)00086-x

Rath, N., and Olson, M. F. (2012). Rho-associated kinases in tumorigenesis: re-considering ROCK inhibition for cancer therapy. EMBO Rep. 13, 900-908. doi: 10.1038/embor.2012.127

Ravanan, P., Srikumar, I. F., and Talwar, P. (2017). Autophagy: The spotlight for cellular stress responses. Life Sci. 188, 53-67. doi: 10.1016/j.lfs.2017.08.029

Roczniak-Ferguson, A., Petit, C. S., Froehlich, F., Qian, S., Ky, J., Angarola, B., et al. (2012). The transcription factor TFEB links mTORC1 signaling to transcriptional control of lysosome homeostasis. Sci. Signal. 5:ra42. doi: 10.1126/scisignal.2002790

Rush, T., Martinez-Hernandez, J., Dollmeyer, M., Frandemiche, M. L., Borel, E., Boisseau, S., et al. (2018). Synaptotoxicity in Alzheimer's disease involved a dysregulation of actin cytoskeleton dynamics through cofilin 1 phosphorylation. J. Neurosci. 38, 10349-10361. doi: 10.1523/JNEUROSCI. 1409-18.2018

Saxton, R. A., and Sabatini, D. M. (2017). mTOR Signaling in growth, metabolism and disease. Cell 168, 960-976. doi: 10.1016/j.cell.2017.03.035

Schaafsma, D., Gosens, R., Zaagsma, J., Halayko, A. J., and Meurs, H. (2008). Rho kinase inhibitors: a novel therapeutical intervention in asthma? Eur. J. Pharmacol. 585, 398-406. doi: 10.1016/j.ejphar.2008.01.056

Scheff, S. W., Price, D. A., Schmitt, F. A., and Mufson, E. J. (2006). Hippocampal synaptic loss in early Alzheimer's disease and mild cognitive impairment. Neurobiol. Aging 27, 1372-1384. doi: 10.1016/j.neurobiolaging.2005.09.012

Scholl, M., Lockhart, S. N., Schonhaut, D. R., O'neil, J. P., Janabi, M., Ossenkoppele, R., et al. (2016). PET imaging of tau deposition in the aging human brain. Neuron 89, 971-982. doi: 10.1016/j.neuron.2016.01.028

Sellers, K. J., Elliott, C., Jackson, J., Ghosh, A., Ribe, E., Rojo, A. I., et al. (2018). Amyloid $\beta$ synaptotoxicity is Wnt-PCP dependent and blocked by fasudil Alzheimers Dement. 14, 306-317. doi: 10.1016/j.jalz.2017.09.008

Settembre, C., Zoncu, R., Medina, D. L., Vetrini, F., Erdin, S., Erdin, S., et al. (2012). A lysosome-to-nucleus signaling mechanism senses and regulates the lysosome via mTOR and TFEB. EMBO J. 31, 1095-1108. doi: 10.1038/emboj.2012.32

Shi, J., Surma, M., Yang, Y., and Wei, L. (2019). Disruption of both ROCK1 and ROCK2 genes in cardiomyocytes promotes autophagy and reduces cardiac fibrosis during aging. FASEB J. 33, 7348-7362. doi: 10.1096/fj.20180 2510R

Shibuya, M., Hirai, S., Seto, M., Satoh, S., and Ohtomo, E. (2005). Effects of fasudil in acute ischemic stroke: results of a prospective placebo-controlled doubleblind trial. J. Neurol. Sci. 238, 31-39. doi: 10.1016/j.jns.2005.06.003

Silva, M. C., Nandi, G. A., Tentarelli, S., Gurrell, I. K., Jamier, T., Lucente, D., et al. (2020). Prolonged tau clearance and stress vulnerability rescue by pharmacological activation of autophagy in tauopathy neurons. Nat. Commun. 11:3258. doi: 10.1038/s41467-020-16984-1

Stranahan, A. M., and Mattson, M. P. (2010). Selective vulnerability of neurons in layer II of the entorhinal cortex during aging and Alzheimer's disease. Neural Plast. 2010:108190. doi: 10.1155/2010/108190

Swanger, S. A., Mattheyses, A. L., Gentry, E. G., and Herskowitz, J. H. (2015). ROCK1 and ROCK2 inhibition alters dendritic spine morphology in 
hippocampal neurons. Cell Logist. 5:e1133266. doi: 10.1080/21592799.2015. 1133266

Tatenhorst, L., Eckermann, K., Dambeck, V., Fonseca-Ornelas, L., Walle, H., Lopes Da Fonseca, T., et al. (2016). Fasudil attenuates aggregation of alphasynuclein in models of Parkinson's disease. Acta Neuropathol. Commun. 4:39. doi: 10.1186/s40478-016-0310-y

Thomanetz, V., Angliker, N., Cloëtta, D., Lustenberger, R. M., Schweighauser, M., Oliveri, F., et al. (2013). Ablation of the mTORC2 component rictor in brain or Purkinje cells affects size and neuron morphology. J. Cell Biol. 201, 293-308. doi: $10.1083 /$ jcb. 201205030

Tonges, L., Gunther, R., Suhr, M., Jansen, J., Balck, A., Saal, K. A., et al. (2014). Rho kinase inhibition modulates microglia activation and improves survival in a model of amyotrophic lateral sclerosis. Glia 62, 217-232. doi: 10.1002/glia. 22601

Tramutola, A., Triplett, J. C., Di Domenico, F., Niedowicz, D. M., Murphy, M. P., Coccia, R., et al. (2015). Alteration of mTOR signaling occurs early in the progression of Alzheimer disease (AD): analysis of brain from subjects with pre-clinical $\mathrm{AD}$, amnestic mild cognitive impairment and late-stage $\mathrm{AD}$. J. Neurochem. 133, 739-749. doi: 10.1111/jnc.13037

Urbanska, M., Gozdz, A., Swiech, L. J., and Jaworski, J. (2012). Mammalian target of rapamycin complex 1 (mTORC1) and 2 (mTORC2) control the dendritic arbor morphology of hippocampal neurons. J. Biol. Chem. 287, 30240-30256. doi: 10.1074/jbc.M112.374405

Wu, J. W., Hussaini, S. A., Bastille, I. M., Rodriguez, G. A., Mrejeru, A., Rilett, K., et al. (2016). Neuronal activity enhances tau propagation and tau pathology in vivo. Nat. Neurosci. 19, 1085-1092. doi: 10.1038/ nn. 4328

Yang, Z., and Klionsky, D. J. (2010). Mammalian autophagy: core molecular machinery and signaling regulation. Curr. Opin. Cell Biol. 22, 124-131. doi: 10.1016/j.ceb.2009.11.014

Conflict of Interest: The authors declare that the research was conducted in the absence of any commercial or financial relationships that could be construed as a potential conflict of interest.

Copyright $\odot 2021$ Weber and Herskowitz. This is an open-access article distributed under the terms of the Creative Commons Attribution License (CC BY). The use, distribution or reproduction in other forums is permitted, provided the original author(s) and the copyright owner(s) are credited and that the original publication in this journal is cited, in accordance with accepted academic practice. No use, distribution or reproduction is permitted which does not comply with these terms. 